\section{A UNIVERSAL EXTENSION-SPLINT FOR GUNSHOT FRACTURES OF THE UPPER LIMB.}

By REGINALD WILLIAMS, L.R.C.P. \& S. EDIN.,

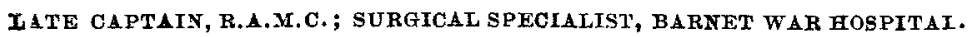

So many are the methods for dealing with fractures of the bones of the arm that it might seem superfluous to suggest another were it not that in that very multiplicity of method a difficulty lies. For though the specialist, particularly if appointed to a hospital in England, may be able to make suitable selection for an individual case from a varied assortment of apparatus, this is impracticable in field-service work, where simplicity and unification are of paramount importance. The question therefore arises, is it possible from a study of the cardinal principles of the already existing types to evolve a simple apparatus for each limb, affording not only adequate treatment for all typical fractures, but easily applied, adjusted, and packed.

In the present article the problem of the upper limb alone is dealt with, consideration of the lower limb being deferred till later. And, first, as to the essential attributes of such a splint. A splint for gunshot fractures should provide: 1. Efficient immobilisation of the whole limb. 2. Ready access to wounds without disturbance of immobilisation. 3. Adequate extension of the broken segments. 4. Maintenance of joints in a position of physiological rest or of

FIG. 1.

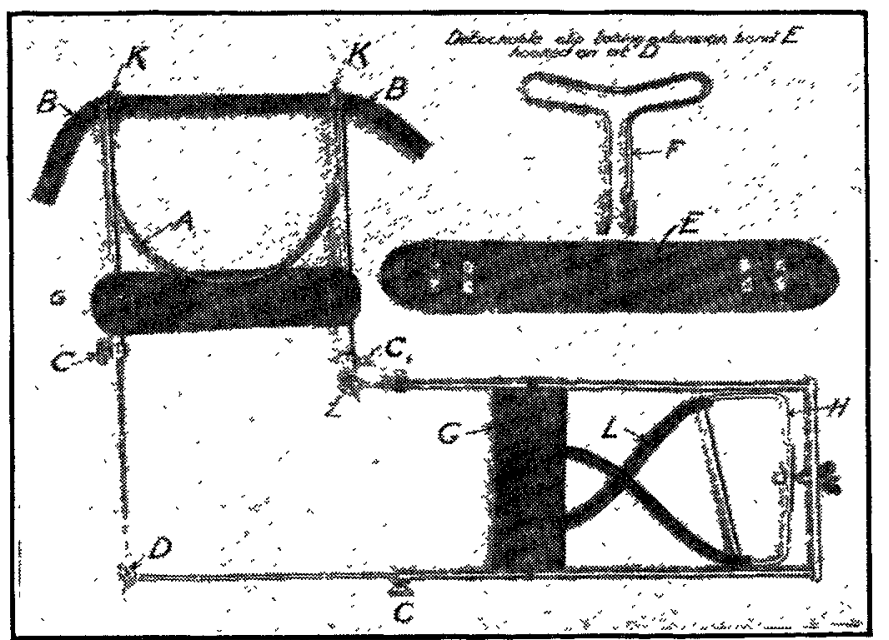

maximum functional utility in view of possible subsequent stiffness and adhesions. 5. Distribution of pressure over as wide an area as possible to prevent pressure sores. 6 . Light. ness and adaptability for application to limb and also for transport. 7. A composition, such as rubber and galvanised metal, which can be easily cleaned and sterilised.

$$
\text { Description of Splint. }
$$

It is probably generally acknowledged that the only system which adequately combines immobilisation with access for dressings is some form of metal frame following the outline of the limb and affording support to the latter by means of slings.

Fig. 1 shows a splint of galvanised wire after the pattern of the cradle splint of Major E. Hey Groves, but differing essentially therefrom in that the wire framework does not pass under the axilla. The shoulder-piece $(A)$ is hinged to the tubular framework in such a manner as to allow of its being swung into any position. It can thus be used for either arm and packed flat for transport. Through the hinges connecting the shoulder-piece to the frame a rubber tubular band passes under the axilla, firmly gripped by the said loops, but not too firmly to prevent adjustment. To the forward end of the splint is affixed an angled loop of wire (II) secured by a bolt which passes through the frame and forms a pivot about which the loop can turn, the bolt being provided with a winged nut by which it can be tightened at will and "set at any desired angle of supination. In whatever way the wrist is fixed, as the hand-piece swivels on a transverse bar movements of flexion of the wrist are not prevented, nor are movements of the fingers interfered with. A flat elastic band $(L)$ is passed under the wrist and crossed over the back of the hand, the hooked end being attached to the angled loop of wire (H). Accessory rubber slings ( $G \mathrm{G}$ ) are hooked on to the frame when necessary. The detachable $T$-shaped metal loop (F) is hooked on to the winged nut (D) at the lower angle, and the broad elastic band (E) is affixed below the elbow for fracture of the humerus (see Fig. 2) and behind the elbow for fractures of the lower arm, as shown in Fig. 4.

FIG. 8.

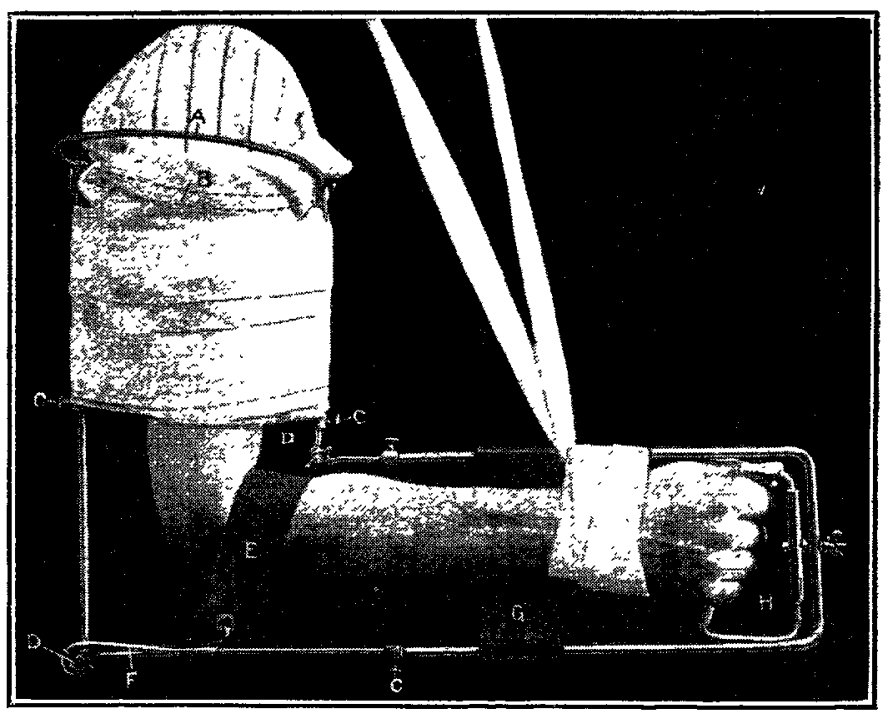

Universal splint applied to a case of fractured humerus.

Method of Application.

Fracture of humerus (Fig. 2).-One end of the axillary tube (B) having been withdrawn, the angled splint is laid over the fractured limb. Accessory rubber slings $(G G)$ are placed in position; the tubular axillary band is replaced in the head-piece and drawn taut, thus fixing the splint at the shoulder. The wrist is next fixed to the hand-piece (H). The broad elastic band $(\mathrm{E})$ is then passed over the forearm and fastened with the necessary degree of tension to the T-shaped metal loop (F). (See Fig. 3.) By this arrangement constriction of the soft parts and interference with the venous return are avoided. Traction being now applied, the fractured segments of bone are drawn asunder, the rubber tube in the axilla pulling upwards and the elastic band downwards.

As to final slinging and adjustment, the three cardinal points of the arm-viz., the shoulder, elbow, and wristhaving been immobilised, dressings are attached to the limb by bandages which encircle the arm and corresponding part of the splint, the removal of which in no way disturbs the adjustment of the fracture. When the patient is up a

Fig. 3.

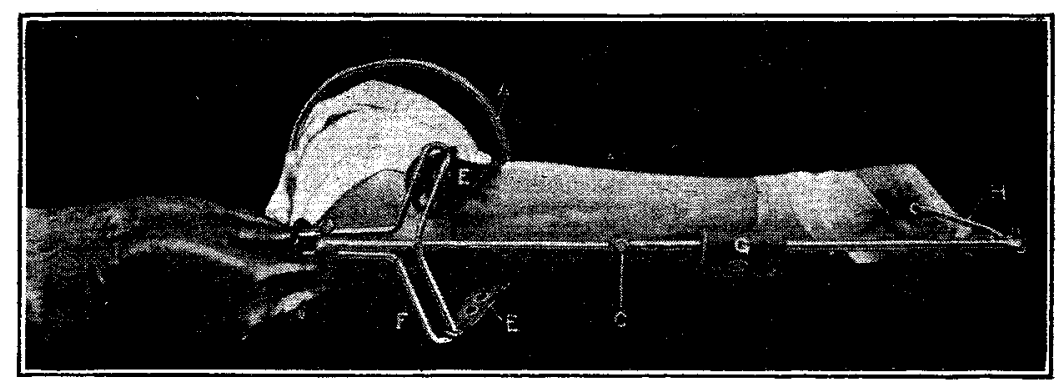

Fractured humerus put up as in Fig. 1, the limb being raised and abducted for dressing purposes.

narrow sling passes round the wrist portion of the splint and supports it to the neck. The limb can be lifted into any position desirable for dressing and irrigation purposes, as also for the prevention of fixation at the shoulder-joint. (See Figs. 2 and 3. )

Fracture of forearm. - The shoulder having been fixed, the rubber band (L) is attached to the hand-piece $(H)$, which is 
fixed in any position desired by means of the $T$ nut controlling the hand-piece. The rubber band $(E)$ is passed above the elbow and attached to the T-shaped metal loop $(\mathbf{F})$, now fixed in position behind the elbow. 'The $T$ screws (C) are loosened and the forward end of the splint is drawn out till sufficient tension on the broken segments is obtained. The screws are then fixed in this position. Fig. 4.)

FIG. 4.

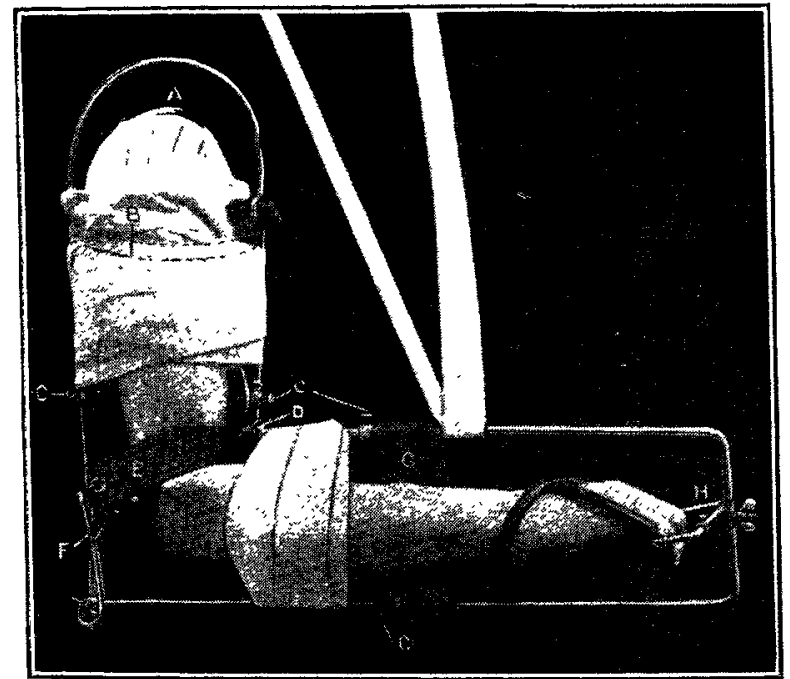

Universal splint applied to a case of fractured forearm. The forearm is completely pronated.

Fracture in elbow region.-In Fig. 5 the $\mathrm{T}$ screws (C C C C) and the winged nuts (D D) (Fig. 1) have been loosened and the forearm portion of the splint has been straightened and fixed in position. The $T$-shaped attachable loop ( $F$ ) has been removed and the rubber band $(E)$ has been used as an accessory sling.

Advantages of the splint.

The advantages of this splint are the following:

1. Only one splint is required for any typical fracture of the upper limb.

2. A gradual, painless, and sufficient extension is effected.

3. It is the ideal splint for field dressing stations. Once affixed, it need never beremoved, as the rubber bands can be detached and sterilised daily.

4. The weight of the splint, with attachments, is only $1 \frac{3}{4} \mathrm{lb}$.

5. It can be packed flat or in sections. By the latter method it can be sent by post in a cardboard box $12 \times 9 \times 1$ in.

6. The entire splint can be sterilised and can at any time be placed in an arm-bath

7. The comfort of the patient is enormously increased and pain is practically eliminated

8. The nurse's work is reduced to a minimum.

This splint is a combination of three splints designed and used by the writer at the Barnet War Hospital, and the results there obtained emboldened him to seek a wider field. They have been tried and approved by the following surgeons, whose encouragement the writer would here wish to acknow-

ledge: Colonel A. Carless, Eastern Command ; Major H. Hey Groves, Beaufort War Hospital ; Surgeon-General N. R. Howse, C.B., V.C., Australian Forces; Captain F. C. Hughes and Sir W. Arbuthnot Lane, Bart., Millbank ; Mr. D. Ligat, Middlesex Hospital ; Mr. V. Warren Low, St. Mary's Hospital ; Colonel T. H. Openshaw, C.M.G., London Hospital. Special indebtedness is due to Major Hey Groves for the inspiration of the work and assistance in preparing thi article; to Lientenant H. T. Booker, R.H., for the drawings ; and to Lientenant W. Daunt, R.A.M.C., High Barnet War Hospital, for experimenting with the earlier models.
ON THE TREATMENT OF

\section{RECENT GUNSHOT WOUNDS WITH} BISMUTH-IODOFORM-PARAFFIN PASTE.

WITH SPECIAL REFERENCE TO COMPOUND FRAC'TURES.

By L. CO L L E D G E, M.B. CaNTab., F.R.C.S. ENG., CAPTAIY, R.A.M.C. ('T.F.);

AND

HAMILTON DRUMMOND, M.B. DURH., F.R.O.S. EDIN., CAPTAIN, R.A.M.C. (T.F.)

A LARGE number of cases have now been reported in which the bismuth-iodoform-paraffin paste (B.I.P.P.), introduced by Professor Rutherford Morison, has been employed with satisfactory results. These cases, however, have all been sent to England suppurating freely before this treatment was applied, and its use in recent wounds has not been reported. The writers wish to record their experience of the use of B.I.P.P. in recent wounds as encountered in a casualty clearing station and to advocate this method as a treatment for recently infected wounds in which suppuration is not yet fully established. They have been further encouraged by the report of Dr. Louisa Garrett Anderson and Dr. Helen Chambers on a large series of cases treated in Fngland, for they say "the best results are obtained when B.I.P.P. treatment is applied soon after the man has been wounded." The danger of gas gangrene has deterred surgeons from employing the method at this early stage in wounds which are constantly infected with the various strains of organisms which produce gas gangrene. Fleming found in the bacteriological examination of 127 cases of wounds from one to seven days old $B$. aerogenes capsulatus ( $B$. perfringens) in 103 cases, streptococci in 102 cases, staphylococci in 40 cases, $B$. tetani in 22 cases. He found also a variety of other organisms in 67 cases. In a series of 25 specimens, including missiles, fragments of clothing, pieces of excised skin and portions of resected muscle from wounds seen in this casualty clearing station within 24 hours of the receipt of the injury, the following organisms were found by Captain J. W. McNee and Captain J. S. Dunn in films and cultures : in 23 cases large bacilli of perfringens type ; in 9 cases streptococci ; in 1 case Staphylococous aureus; in 1 case Gram-negative bacilli. In only one instance in which some fragments of cloth from a wound were examined by films and cultures was the result negative.

\section{Advantages of Method and Technique.}

The method rightly used has the following advantages :-

1. The wound can be closed by sutures, rendering prolonged drainage unnecessary, and in the great majority of cases there is no suppuration.

2. No change of dressings is required and much disturbance of the patient is avoided - a most important matter to freshly wounded men to whom rest is essential.

3. It greatly increases the facility and reduces the discomfort of transport. Apart from wounds of the viscera and central nervous system, the management of compound fractures presents the most difficult problem for the surgeon in a casualty clearing station, and it is in this class of cases that the writers have found the method particularly valuable.

4. There is no increased liability to the development of gas gangrene. On the contrary, it is diminished.

Technique.-The patients usually arrive in a filthy condition, encrusted with mud. The skin around the wounds, for now they are usually multiple shell, bomb, or grenade wounds, is cleaned with ether soap and rendered as surgically clean as possible. The part is surrounded with dry sterilised towels. Surrounding the entry and exit wounds there is always a margin of necrotic skin heavily infected with micro-organisms. This is removed by excising a ring of skin one-eighth of an inch wide. The wound is then freely opened up and all foreign bodies and loose pieces of bone are as far as possible removed. Further, all devitalised muscle is resected and bleeding points are secured with Spencer-Wells artery forceps. This resection extends not only to mnscle which is obviously necrotic, but also to areas which are devascularised and non-contractile. It is in muscle in this condition that gas gangrene begins to spread. Although this procedure is most important, the writers have sufficient to prevent the subsequent development of obvious signs of infection in compound fractures. The raw surface 